\title{
Whole genome, exon mutation and transcriptomic profiling of acute myeloid leukemia: A case report
}

\author{
SI-HAN LAI ${ }^{*}$, YE-CHENG LI* , SHAN ZHANG, RUI DENG, YAN DENG and FANG-YI FAN \\ Hematology Department and Hematopoietic Stem Cell Transplantation Center, \\ The General Hospital of Western Theater Command, Chengdu, Sichuan 610083, P.R. China
}

Received March 19, 2020; Accepted December 14, 2020

DOI: $10.3892 / \mathrm{ol} .2021 .12820$

\begin{abstract}
The present study aimed to observe previously unidentified gene mutation and expression profiles associated with acute myeloid leukemia (AML) at the individual level, based on the blood samples of a father-son pair. Genomic DNA and RNA samples from blood serum were collected. Whole-genome sequencing (WGS) and whole-exome sequencing (WES), as well as mRNA sequencing of the son, were performed. For the father's sample, a total of 3,897,164 single nucleotide polymorphisms (SNPs) and 780,834 insertion and deletions (indels) were identified. Regarding amino acid translation, there were 11,316 non-synonymous, 12 stop-loss, 12,033 synonymous, 92 stop-gain SNPs, 63 frameshift insertions, 73 frameshift deletions, 242 non-frameshift insertions, 248 non-frameshift deletions, four stop-gains and two stop-loss for indel variants. Among the AML-related genes that had been previously identified, 14 genes were found in the father's exon region. For WES of the son's DNA, 96,639 SNPs were identified, including 10,504 non-synonymous SNPs. Seven mutant genes were found in sons' exon region compared with 121 AML-related genes. Based on the transcriptomic sequencing, there were 54 differentially expressed mRNAs, including 31 upregulated and 23 downregulated mRNAs. In the exon region, 10,072 SNPs were detected, and different types of alternative splicing in the son's sample were observed. Overall, whole genome, exon mutation and transcriptomic profiling of the present two patients with AML may provide a new insight into the molecular events governing the development of AML.
\end{abstract}

Correspondence to: Dr Fang-Yi Fan, Hematology Department and Hematopoietic Stem Cell Transplantation Center, The General Hospital of Western Theater Command, 270 Rong Du Avenue, Chengdu, Sichuan 610083, P.R. China

E-mail: 834525469@qq.com

*Contributed equally

Key words: acute myeloid leukemia, whole-genome sequencing, whole-exome sequencing, transcriptomic sequencing, Fms related receptor tyrosine kinase 3

\section{Introduction}

Acute myeloid leukemia (AML) is the most common acute leukemia in adults and one of the most common hematological malignancies, with strong biological heterogeneity and clinical heterogeneity (1). It accounted for $20 \%$ of acute leukemia cases in children worldwide in 2008 (1). Due to chromosomal abnormalities and genetic mutations, the normal hematopoietic system undergoes malignant transformation at different stages, which causes blockade of blood cell differentiation and results in different subtypes of AML in the process of directional myeloid differentiation (2). An important pathogenic factor of AML is chromosomal abnormality, including translocation, inversion, deletion and tandem duplication (3). There are varieties of recurrent genetic abnormalities, such as $\mathrm{t}(8: 21)$, inv (16) and $\mathrm{t}$ (15:17) (4). In addition to large chromosomal abnormalities, genetic mutation is another feature for AML (5). With the development and widespread application of high-throughput sequencing technologies, leukemia-associated mutant genes have been identified with diagnostic and therapeutic values in AML. A few gene mutations, including Fms related receptor tyrosine kinase 3 (FLT3), nucleophosmin 1, KIT proto-oncogene, receptor tyrosine kinase, CCAAT enhancer binding protein $\alpha$, tet methylcytosine dioxygenase 2 , and DNA methyltransferase $3 \alpha$ have been associated with AML (6,7). At the individual level, more previously unknown gene mutations associated with AML should be identified. The present study reported two AML cases with kinship (a father-son pair) in which genome-wide sequencing, whole-exome sequencing (WES) and transcriptomic analysis was performed, and some novel mutant genes and sites were identified.

\section{Materials and methods}

Patients. A father-son pair were both diagnosed with AML at The General Hospital of Western Theater Command (Chengdu, China). The son was diagnosed in April 2014 while the father was diagnosed in May 2016. The father was aged 66 and the son aged 44 years. According to National Comprehensive Cancer Network Guideline for AML (8), both patients were diagnosed with AML and vein blood samples were collected, and DNAs were extracted for whole-genome sequencing (WGS) or WES using the phenol-chloroform 
method. The Hospital Ethics Committee approved the study and the two patients signed informed consent before donating samples. For transcriptome sequencing, the RNA sample was extracted from the blood of the son.

$W G S$. The DNA sample from the father was used for genome-wide sequencing. DNA was extracted from peripheral white blood cells using a DNA extraction kit (Qiagen $\mathrm{GmbH})$ according to the manufacturer's instructions. A total of $2 \mu \mathrm{l}$ purified DNA was used to measure the quality and quantity of the DNA (ng/ $\mu$ l) (A260/A280) using an UV visible spectrophotometer (BioTek Instruments, Inc.). Finally, $20 \mathrm{ng} / \mu \mathrm{l}$ DNA samples were stored at $20^{\circ} \mathrm{C}$, until further use. DNA degradation and the molecular weight was analyzed using $0.2 \%$ agarose gel electrophoresis (loading $5 \mu 1$ extracted DNA). DNA libraries were prepared following the manufacturer's protocol (Illumina DNA Prep with Enrichment; cat. no. 20025523; Illumina, Inc.). Briefly, for each sample, DNA was firstly sheared into fragments of $\sim 350 \mathrm{bp}$. The fragments were then end-repaired, A-tailed, ligated to paired-end adaptors and PCR amplified (according to the manufacturer's instructions) for library construction. The final library concentration used was $6.2 \mathrm{pM}$. The resulting DNA libraries were subjected to 150 bp pair-end sequencing on the Illumina HiSequencing PE150 platform (Illumina, Inc.). The reference genome was downloaded from the University of California, Santa Cruz (UCSC) database (GRCh38/hg38; http://genome.ucsc.edu). Low-quality reads were discarded using the PRINSEQ software (v0.20.4) and the resulting clean data were aligned to the human reference sequence (hg19) with the Burrow-Wheeler Aligner (BWA) software (0.7.12-r1044) (9). Duplicate reads were removed using the Picard software (http://sourceforge. net/projects/picard/). The aligned reads were sorted with the Genome Analysis Toolkit (GATK) software (v2.8.1; https://github.com/RRafiee/GenomeAnalysisToolkit). SIFT (sift.jcvi.org) and PolyPhen2 (genetics.bwh.harvaed.edu) software were used to predict the effects of amino acid mutations on protein structure and function. All variants were annotated with ANNOVAR (10).

WES. The DNA sample from the son was used for WES. The protocol of DNA extraction was identical to that of the father's sample. DNA was randomly digested into 150-200 bp fragments using a Bioruptor Pico ultrasound. Fragmented DNA was end-repaired, A-tailed and then connected. Sample labeling and enrichment of DNA were conducted using PCR amplification according to the manufacturer's instructions. The DNA library with the specific index was subjected to liquid phase hybridization with the biotin-labeled RNA probe, and the target gene exon was obtained using the streptavidin-labeled magnetic beads. Then, target exon genes were enriched by PCR amplification. Sequencing was performed using an Ilumina platform (AmpliSeq ${ }^{\mathrm{TM}}$ Focus Panel for Illumina; cat. no. 20019164; $10 \mathrm{ng}$ ) and the HiSeq 3000/4000 PE (150 paired-end) Cluster kit (both from Illumina, Inc.), according to the manufacturer's instrustions. Clean reads were mapped to the reference genome, GRCh37 using BWA. After removing duplications, single nucleotide polymorphisms, and insertions and deletions (Indel) were assigned and annotated using the (GATK) based on dbSNP build 150 .
Table I. Summary of the WGS and WES.

\begin{tabular}{lcr}
\hline Index & WGS & \multicolumn{1}{c}{ WES } \\
\hline Raw reads & $638,386,172$ & $638.39^{\text {a }}$ \\
Raw data & $96,396,311,972$ & $96,396.31^{\text {b }}$ \\
Clean reads & $91,827,913,399$ & $609.51^{\text {a }}$ \\
Mean depth, $x$ & 25 & 28.61 \\
Coverage, $\%$ & 96.90 & 99.91 \\
\hline
\end{tabular}

${ }^{a} \mathrm{M}$. ${ }^{\mathrm{b}} \mathrm{Mb}$. WGS, whole-genome sequencing; WES, whole-exome sequencing.

Transcriptome sequencing. The total RNA from blood samples of the son was extracted using TRIzol ${ }^{\circledR}$ reagent (Invitrogen; Thermo Fisher Scientific, Inc.) according to the manufacturer's instructions. RNA purity was determined using a NanoDrop 2000 spectrophotometer (NanoDrop Technologies; Thermo Fisher Scientific, Inc.), and RNA integrity was verified using 1.5\% agarose gels. Magnetic beads conjugated with oligo (dT) were used to isolate mRNA, which was fragmented into short lengths of 200 bp using an RNA Fragmentation kit (Illumina, Inc.). The short RNA fragments were used as templates for first-strand DNA synthesis with random primers according to the manufacturer's instruction. Next, second-strand DNA was generated, purified using magnetic beads, end repaired, and a single adenine (A) nucleotide was added to the 3 ' ends. DNA degradation and the molecular weight were analyzed using $0.2 \%$ agarose gel electrophoresis (loading $5 \mu \mathrm{l}$ extracted DNA). RNA-Sequencing (RNA-Seq) libraries were prepared using the VAHTS Total RNA-seq (H/M/R) Library Prep kit for Illumina (Vazyme Biotech Co., Ltd.) following the manufacturer's instructions. The raw reads were processed to remove the adapter and primer sequences using SeqPrep software (v1.0; https://github.com/jstjohn/SeqPrep). Low-quality bases at the 3 ' ends were deleted using Sickle software (v1.33; https://github.com/najoshi/sickle). Fragments $<30 \mathrm{bp}$ in length were excluded from further analyses and reads containing $\mathrm{N}>10 \%$ were removed. The obtained high-quality sequencing sequence after quality control was aligned with the designated Ensembl genome using TopHat (http://tophat.cbcb.umd.edu/). Saturation, duplicate reads and coverage analysis were conducted software from Majorbio, including SeQC-2.3.2 (http://code.google.com/p/rseqc/) and RSeQC-2.3.2 (http://code.google.com/p/rseqc/). Predictionof novel transcripts in annotated genomes was performed using Cufflink (http://cufflinks.cbcb.umd.edu/) (11). To identify the known long non-coding (lnc)RNAs, the predicted novel transcript was aligned with a known lncRNA using collection of databases, such as NONCODE (www.noncode.org), Ensembl (ensembl.org), NCBI (http://www.ncbi.nlm.nih. gov/), and UCSC (genome.ucsc.edu), LncRNAdb (http://www. lncrnadb.org/), GENCODE (https://www.gencodegenes. org/) and LncRNA Disease. The gene expression levels and differentially expressed genes (DEGs) were estimated using cufflinks (http://cole-trapnell-lab.github.io/cufflinks/) (12-14). The default parameters for DEGs were false discovery rate (FDR) $\leq 0.05$ and $\log 2$ fold-change (FC) $\geq 1$ or $\leq-1$. 

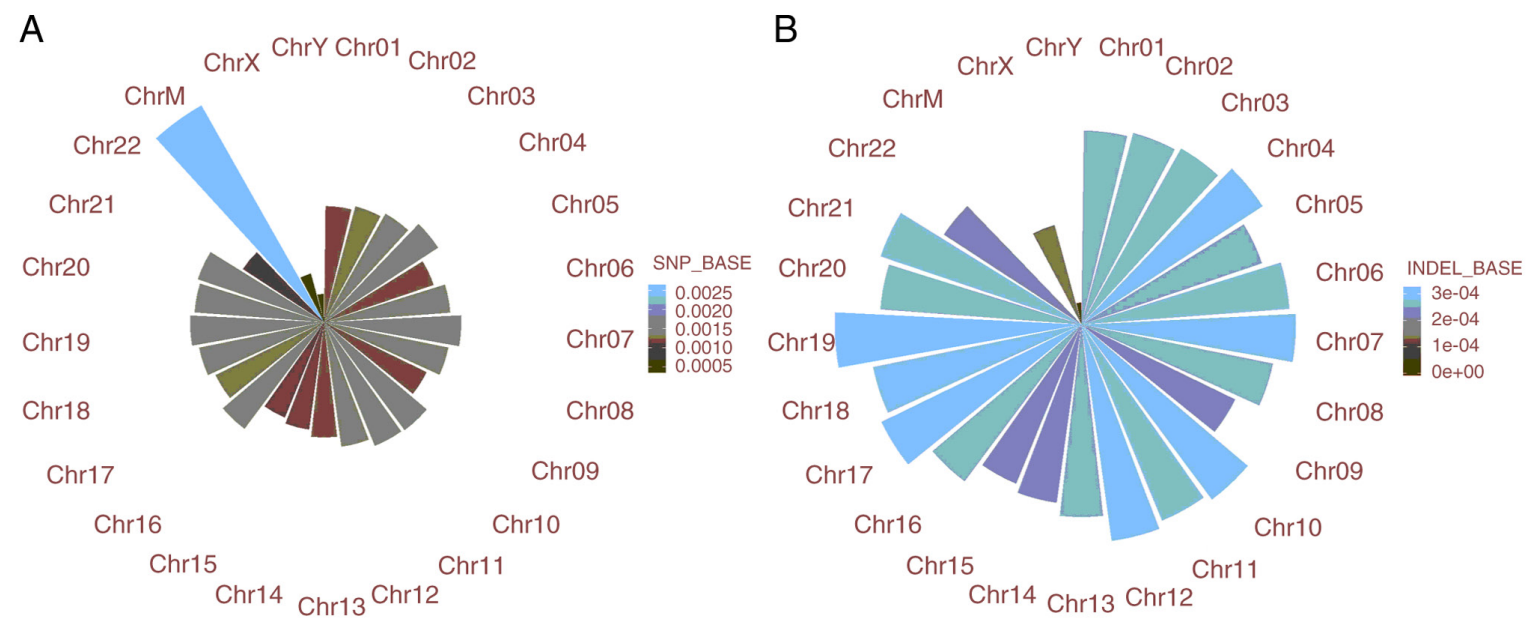

Figure 1. Distribution of SNPs and indels in different chromosomes. (A) SNP base distribution. (B) Indel base distribution. SNP, single nucleotide polymorphism; indel; insertion deletion.
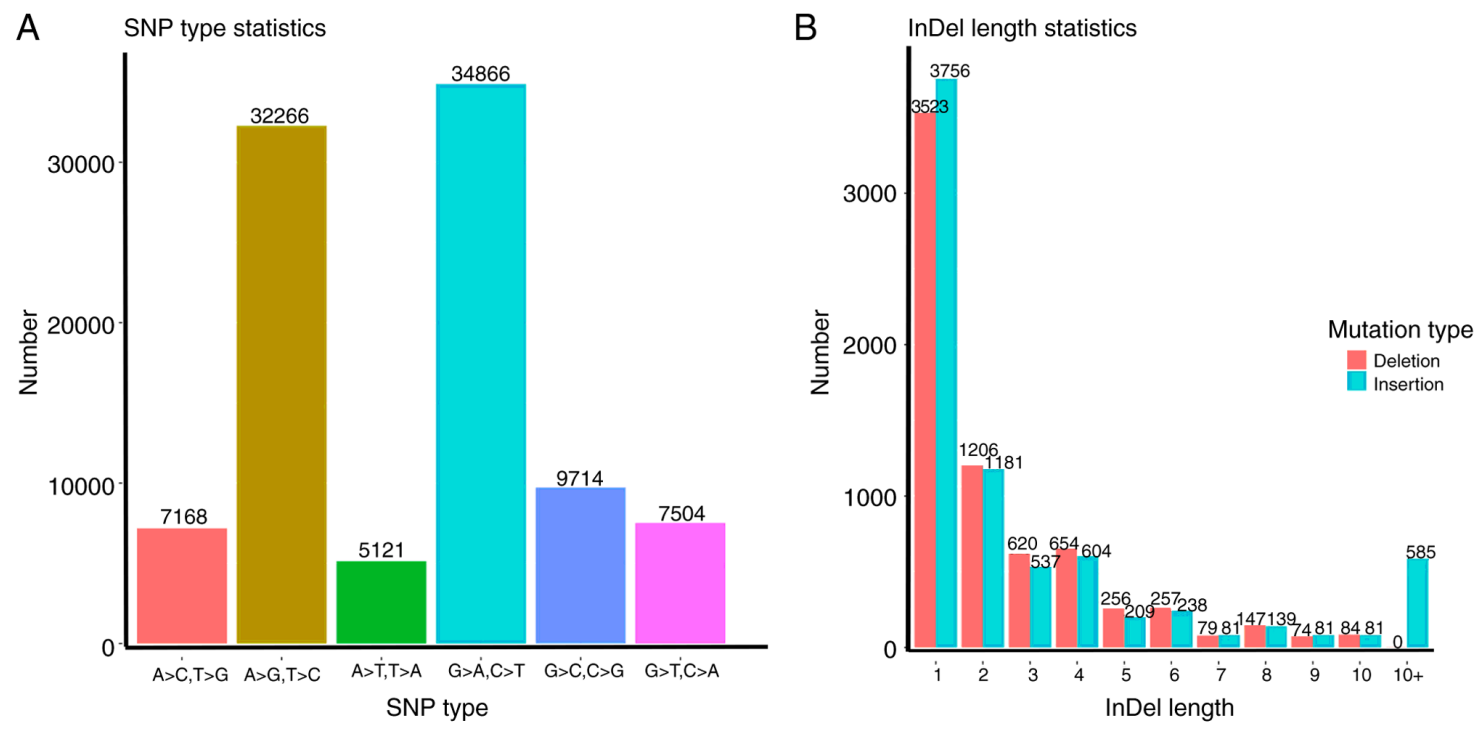

Figure 2. SNP types and distribution of indel lengths. (A) SNP types and (B) distribution of indel lengths. SNP, single nucleotide polymorphism; indel; insertion deletion.

Gene Ontology (GO) enrichment analysis was performed using the software Goatools (https://github.com/tanghaibao/GOatools). The P-values were corrected using four multiple test methods (Bonferroni's, Holm, Sidak and FDR) to control the calculated false positive rate. When the corrected P-value (or P-FDR) $\leq 0.05$, it was considered significant (15-17). Kyoto Encyclopedia of Genes and Genomes (KEGG) pathway enrichment analysis was performed using KOBAS (http://kobas.cbi.pku.edu.cn/home.do). A corrected $\mathrm{P} \leq 0.05$ was considered to indicate a significant difference (18). Samtools (http://samtools.sourceforge.net/) and VarScan version 2.2.7 (http://varscan.sourceforge.net/) software were used to find candidate SNPs.

\section{Results}

WGS results. The WGS results for the father's blood sample were as follows. A total of 638,386,172 raw reads were generated as shown in Table I, achieving WGS mean depth of 25 .
The coverage of the whole genome was $96.9 \%$. All properly pair-end mapped sequences were used for subsequent variants detection. A total of 3,897,164 SNPs and 780,834 indels were identified (Fig. 1). The distribution of each SNP locus was analyzed (Fig. 2A) (Table II). The results showed that $\mathrm{C}>\mathrm{T}$ and $\mathrm{G}>\mathrm{A}$ were the most common variations. The distribution of indel lengths is presented in Fig. 2B. All SNP and indel variants were annotated using the ANNOVAR program in conjunction with the reference gene annotation information of the UCSC Genome Browser. A total of 24,150 SNPs located in the exon region were probed and 727 indel variants in the exon region were found. The distribution of SNPs and indel variants in gene functional regions shown in Table II.

Regarding amino acid translation, there were 11,316 non-synonymous, 12 stop-loss, 12,033 synonymous, 92 stop-gain for SNPs, 63 frameshift insertions, 73 frameshift deletions, 242 non-frameshift insertions, 248 non-frameshift deletions, 4 stop-gain, 2 stop-loss for indel variants (Table III). 
Table II. Acute myeloid leukemia-related mutation genes in the exon region from father's whole-genome sequencing results.

\begin{tabular}{|c|c|c|c|c|c|c|c|c|}
\hline Chrom & Position & Refs. & Alt & Gene & Transcript & $\begin{array}{l}\text { Exon } \\
\text { region }\end{array}$ & $\begin{array}{l}\text { Variant } \\
\text { transcript }\end{array}$ & $\begin{array}{l}\text { Variant } \\
\text { protein }\end{array}$ \\
\hline \multirow[t]{3}{*}{10} & 100219374 & $\mathrm{~T}$ & A & HPSE2 & NM_001166245 & 10 & c.A1400T & p.Y467F \\
\hline & & & & & NM_001166244 & 11 & c.A1562T & p.Y521F \\
\hline & & & & & NM_021828 & 12 & c.A1736T & p.Y579F \\
\hline 10 & 104934709 & $\mathrm{~T}$ & $\mathrm{C}$ & NT5C2 & NM_001134373/NM_012229 & $2 / 3$ & c.A7G & p.T3A \\
\hline 10 & 5136651 & $\mathrm{C}$ & G & AKR1C3 & NM_001253909/NM_003739 & 1 & c.C15G & p.H5Q \\
\hline 10 & 5139685 & G & A & AKR1C3 & $\begin{array}{c}\text { NM_001253908/NM_001253909/ } \\
\text { NM_003739 }\end{array}$ & 3 & c.G312A & p.K104K \\
\hline 10 & 54531235 & $\mathrm{C}$ & $\mathrm{T}$ & MBL2 & NM_000242 & 1 & c.G161A & p.G54D \\
\hline 10 & 70405855 & A & G & TET1 & NM_030625 & 4 & c.A3369G & p.I1123M \\
\hline \multirow[t]{2}{*}{10} & 88422116 & $\mathrm{C}$ & $\mathrm{T}$ & OPN4 & NM_033282 & 4 & c.C1181T & p.T394I \\
\hline & & & & & NM_001030015 & 9 & c.C1214T & p.T405I \\
\hline 10 & 89623716 & $\mathrm{G}$ & A & PTEN & NM_001304717 & 1 & c.G10A & p.G4R \\
\hline 10 & 89623901 & G & $\mathrm{C}$ & PTEN & NM_001304717 & 2 & c.G194C & p.C65S \\
\hline 10 & 89624218 & $\mathrm{C}$ & G & PTEN & NM_001304717 & 2 & c.C511G & p.L171V \\
\hline \multirow[t]{3}{*}{11} & 102595492 & G & A & MMP8 & NM_002424 & 1 & c.C95T & p.T32I \\
\hline & & & & & NM_002424 & 2 & c.A259G & p.K87E \\
\hline & & & & & NM_001304441/NM_001304442 & 3 & c.A190G & p.K64E \\
\hline \multirow[t]{2}{*}{11} & 35226155 & A & G & CD44 & NM_001001389 & 9 & c.A1121G & p.K374R \\
\hline & & & & & NM_000610 & 10 & c.A1250G & p.K417R \\
\hline \multirow[t]{3}{*}{11} & 35229673 & $\mathrm{~T}$ & $\mathrm{C}$ & CD44 & NM_001001390 & 6 & c.T689C & p.I230T \\
\hline & & & & & NM_001001389 & 11 & c.T1307C & p.I436T \\
\hline & & & & & NM_000610 & 12 & c.T1436C & p.I479T \\
\hline 13 & 28624294 & G & A & FLT3 & NM_004119 & 6 & c.C680T & p.T227M \\
\hline
\end{tabular}

Chrom, chromosome; ref, reference genome allele; Alt, alternate non-reference allele.

Table III. Summary of detected SNPs and indels in all sequencing samples.

\begin{tabular}{lrr}
\hline A, SNPs & & \\
\hline Index & WGS results & WES \\
\hline Total SNPs in exon & 24,150 & 22,656 \\
Synonymous SNP & 12,033 & 11,570 \\
Non-synonymous SNP & 11,316 & 10,504 \\
Stop-gain & 92 & 87 \\
Stop-loss & 12 & 12 \\
\hline
\end{tabular}

$\mathrm{B}$, Indels

\begin{tabular}{lrr}
\hline Index & WGS & WES \\
\hline Total indels in exon & 727 & 620 \\
Frameshift insertion & 63 & 102 \\
Frameshift deletion & 73 & 117 \\
Non-frameshift insertion & 242 & 156 \\
Non-frameshift deletion & 248 & 190 \\
Stop-gain & 4 & 3 \\
Stop-loss & 2 & 1 \\
\hline
\end{tabular}

SNP, single nucleotide polymorphism; indel, insertion deletion; WGS, whole-genome sequencing; WES, whole-exome sequencing.
Non-synonymous SNPs can cause changes in the encoded amino acids. Together, 2,436 mutations were destructive to protein structure and function according to SIFT, and 1,154 mutations were probably harmful according to PolyPhen2. Comparing with 121 AML-related genes identified in previous AML studies, 14 genes were found in the father's exon region, including the following genes: HPSE2 (19), NT5C2 (20), AKR1C3 (21), MBL2 (22), ARID5B (23), TET1 (24), WT1 (25), CD44 (26) and FLT3 (27). However, only 10 of these had non-synonymous mutations changing encoded amino acids.

WES results. The WES results from the son achieved 96,396.31 Mb raw data, generating a mean depth of 28.61. The coverage rate was $99.91 \%$. All properly pair-end mapped sequences were used for subsequent variants detection, as shown in Table II. Overall, 96,639 SNPs were identified, including 10,504 non-synonymous SNPs. For the types of base mutations in SNPs, $\mathrm{C}>\mathrm{T}$ and $\mathrm{G}>\mathrm{A}$ were the most commonly observed in this sample. The indel results showed 15,033 mutations, including 102 frameshift insertions, 117 frameshift deletions, 156 non-frameshift insertions, 190 non-frameshift deletions, three stop-gain and one stop-loss (Table III). Seven mutant genes were found in son's exon region compared with 121 AML-related genes, including FLT3, GATA2 $(28,29)$, MCM7 (30), PTEN (31), and RUNX1T1 (32) (Table IV). Among them, six genes showed non-synonymous SNPs (Table IV). 
Table IV. Acute myeloid leukemia-related mutation genes in the exon region from son's whole-exome sequencing results.

\begin{tabular}{ccccccccc}
\hline Chrom & Position & Refs. & Alt & Gene & Transcript & $\begin{array}{c}\text { Exon } \\
\text { region }\end{array}$ & $\begin{array}{c}\text { Variant } \\
\text { transcript }\end{array}$ & $\begin{array}{c}\text { Variant } \\
\text { protein }\end{array}$ \\
\hline 1 & 110882830 & C & T & RBM15 & NM_001201545/NM_022768 & 1 & c.C803T & p.P268L \\
3 & 128202797 & C & T & GATA2 & NM_001145662/NM_032638 & 4 & c.G923A & p.R308Q \\
7 & 99696316 & G & A & MCM7 & NM_001278595/NM_182776 & 5 & c.C77T & p.P26L \\
& & & & & NM_005916 & 6 & c.C605T & p.P202L \\
8 & 93107611 & G & A & RUNX1T1 & NM_001198634/NM_001198679 & 1 & c.C85T & p.R29W \\
10 & 89623901 & G & C & PTEN & NM_001304717 & 2 & c.G194C & p.C65S \\
13 & 28609806 & T & G & FLT3 & NM_004119 & 12 & c.A1423C & p.T475P \\
13 & 28674628 & T & C & FLT3 & NM_004119 & 1 & c.A20G & p.D7G \\
\hline
\end{tabular}

Chrom, chromosome; ref, reference genome allele; Alt, alternate non-reference allele.

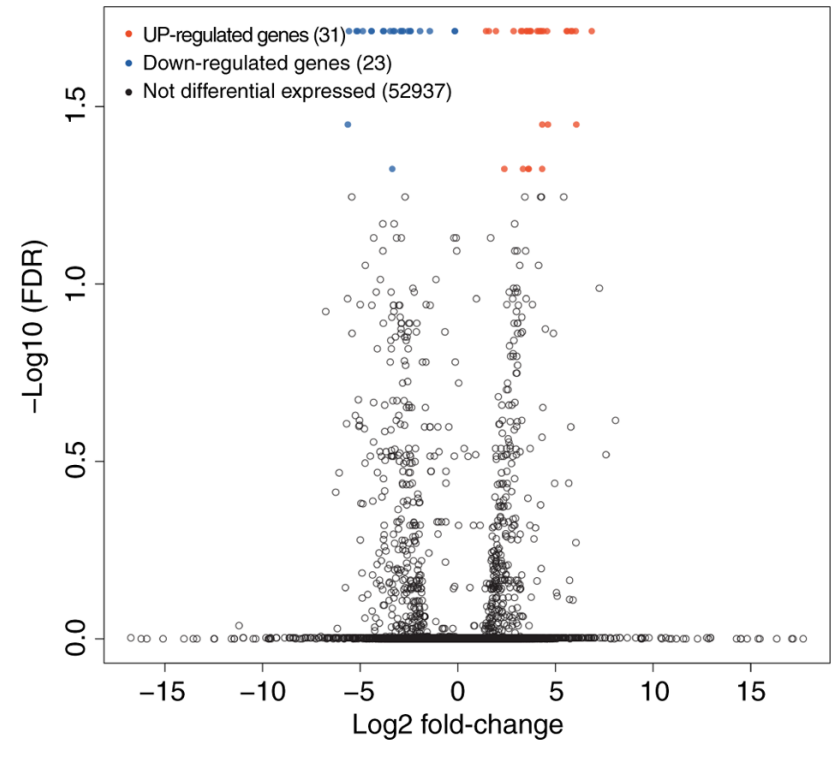

Figure 3. Differentially expressed mRNAs in the transcriptomic analysis.

Then the mutant genes in the exon region were compared between the father and son results, and some common ones were observed. Notably, two missense mutations in the FLT3 gene were identified. Internal tandem duplications in the FLT3 gene (FLT3-ITD) were the earliest described molecular alterations in AML (33). In the present results, the alterations of FLT3 were as follows. In WGS data from the father: Exon 6, c.C680T: p.T227M (NM_004119). In WES data from the son: Exon 1, c.A20G: p.D7G (NM_004119) and exon 12, c.A1423C: p.T475P (NM_004119). Another common gene was PTEN. As Tables III and IV show, in WGS data from the father, the following mutations were identified: c.G10A: p.G4R (NM_001304717) In exon 1, c.G194C: p.C65S(NM_001304717) in exon 2 and c.C511G: p.L171V (NM_001304717) in exon 2. In WES data from the son the c.G194C: p.C65S (NM_001304717) mutation was identified in exon 2.

Transcriptomic sequencing results. Transcriptomic sequencing generated $69,537,564$ raw reads containing
$10,430,634,600$ nucleotides for the son. Following clean-up and quality filtering, 68,019,440 and 75,105,808 clean reads were obtained, with a Q20 percentage (proportion of nucleotides with quality value $>20$ ) $>98.51 \%$, which indicated the RNA sequencing results were of high quality and suitable for use in further analysis.

As Fig. 3 shows, there were 54 differentially expressed mRNAs from transcriptomic analysis, of which 31 were significantly upregulated in AML, and 23 were significantly downregulated. However, no common differentially expressed mRNAs between WGS and WES were identified. As aforementioned, FLT3 and PTEN might play an important role in AML development. Compared with patients with AML, expression of FLT3 was decreased ( $\mathrm{P}=0.035$; FDR, 0.919; $\log 2 \mathrm{FC}, 3.17)$. A lower expression of PTEN were found in the patients with AML without statistical significance $(\mathrm{P}=0.4135$; FDR, 0.993; $\log 2 \mathrm{FC},-0.71)$.

GO annotations revealed that the aforementioned genes were enriched in 'single-organism signaling', 'response to stimulus', 'biological regulation' and 'metabolic process'. The top 30 terms were all among the biological process class and are presented in Fig. 4. KEGG pathway analysis revealed 56 related pathways, while according to corrected P-value, only three pathways were significantly enriched: 'Influenza A', 'hematopoietic cell lineage' and 'cell adhesion molecules' (Fig. 5). Alternative splicing is an important mechanism for regulating gene expression and producing protein diversity, which can cause large differences in genes and proteins (12). There were 4,370 $3^{\prime}$ end alternative splicing events, 2,575 3' untranslated region (UTR) alternative splicing events, 4,927 5' end alternative splicing events, 1,783 5' UTR alternative splicing events, 2,468 exon spanning events, 336 intron retentions, and 2,761 other alternative splicing.

\section{Discussion}

Clinically, it is rare to identify AML cases similar to familial aggregation. In the present study, the common molecular mutation types of AML in the father were not consistent with those of his son. Therefore, it was hypothesized that the father had some molecular variation that had not been previously recognized, which led to the occurrence of AML to the 


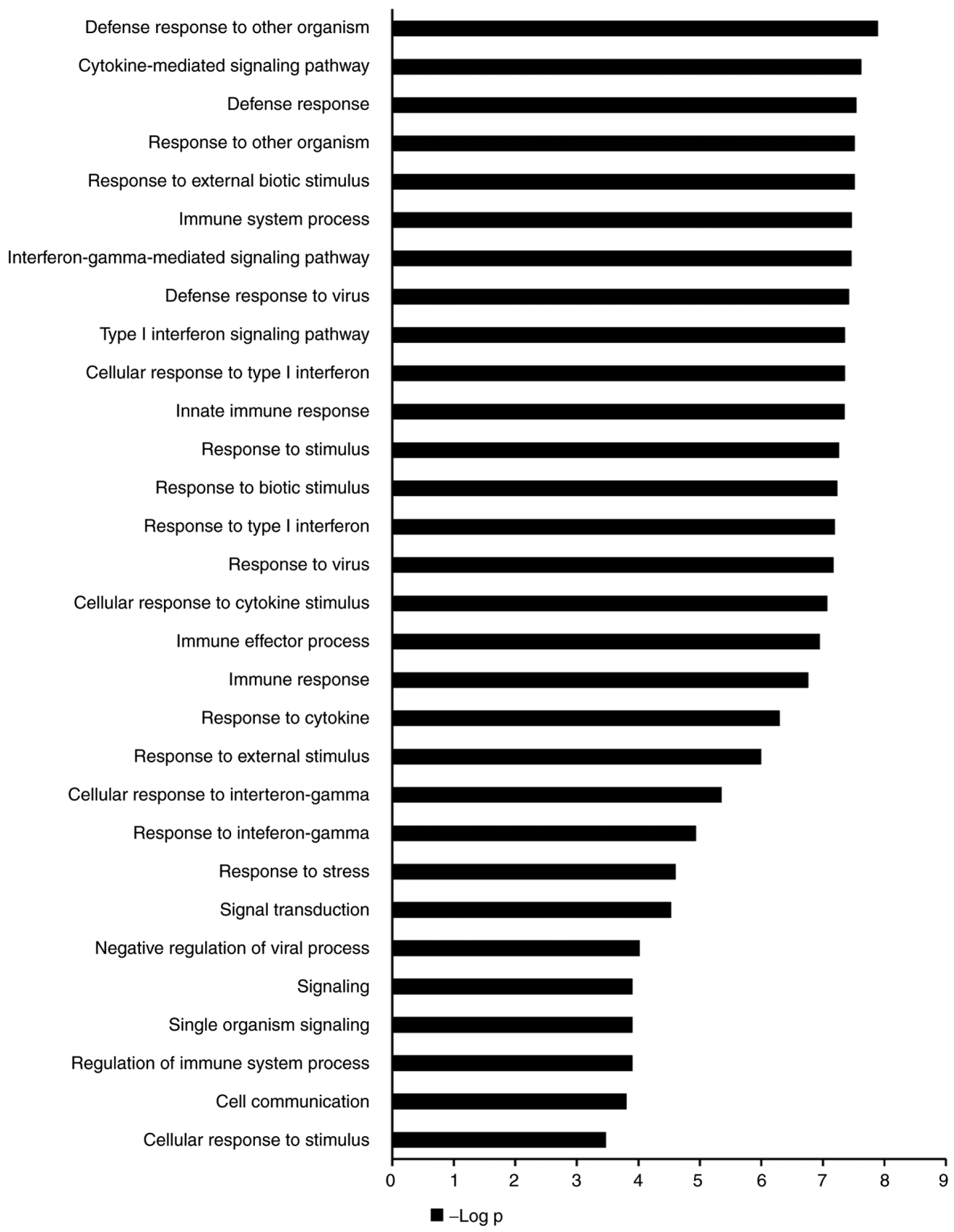

Figure 4. Top 30 terms of Gene Ontology functional enrichment in the transcriptomic analysis.

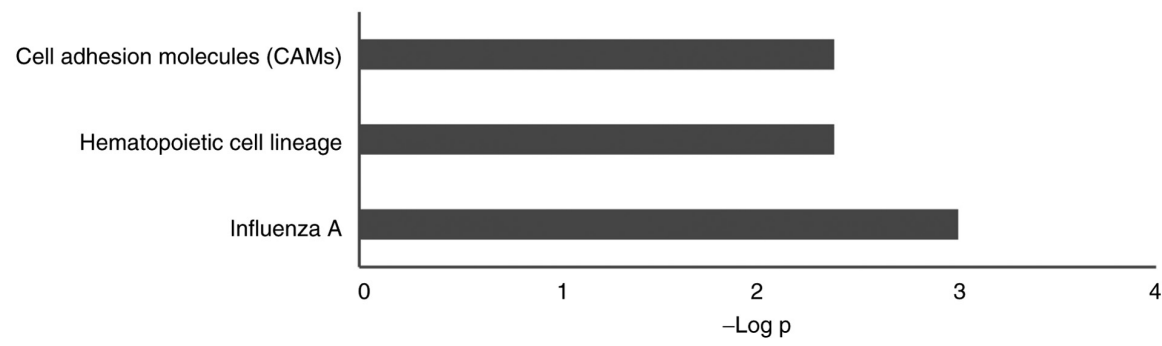

Figure 5. There were 3 pathways are significantly enriched in Kyoto Encyclopedia of Genes and Genomes pathway analysis based on transcriptomic analysis.

father, and it could be stably and continuously expressed in his whole sexual maturity stage. The molecular variation was passed on to his son through genetic material and expressed at a certain developmental stage of the son, leading to the disease. Therefore, the current study performed genome-wide sequencing, WES and transcriptomic analyses of two patients 
with AML patients with kinship (a father-son pair) in order to investigate the profiling of AML development.

The father-son pair samples had a similar number of SNPs and indel variants in the exon region (24,150 SNPs and 727 indel variants in father sample; 22,656 SNPs and 620 indel variants in son sample), though different sequencing analysis technologies were used, following the decision of the patient. Their SNPs and indel variants were similar to previous studies of other types of blood cancer (e.g. chronic myelomonocytic leukemias) $(7,34)$. However, transcriptomic analysis was also performed on the son sample and 10,072 SNPs in the exon region were found.

In total, $>200$ common exon mutant genes were identified in both the father and son, which suggested that AML may be the result of a combination of multiple genes. In these genes, an FLT3 mutation was identified in both father and son. They showed different variations in the exon region. However, different variations in FLT3 may cause the same result. FLT3-ITD has major clinical implications and is associated with adverse outcomes in recurrent somatic mutations in AML through multiple mechanisms (35). Previous studies revealed FLT3-ITD with different insertion sites spread over the whole stretch of exons 14-15 $(36,37)$. However, the present study observed three mutations in exons 1, 6 and 12 and none of these were located in exons 14 and 15 . These mutations may cause changes in protein structure or function, and the further study can investigate the role of these exons. FLT3 is known to be overexpressed in hematopoietic neoplasms (38). The current transcriptomic sequencing results also showed increased expression of FLT3 in AML. PTEN, a tumor suppressor gene (31), was another identified common gene. A missense mutation in the PTEN gene was reported in concurrent germ cell tumor (GCT)-associated AML (39). The mRNA and protein levels of PTEN in newly diagnosed patients with AML and patients with relapsed AML are significantly lower compared with those in the control and remission groups, which was supports the present findings (39).

Through high-throughput analysis, the mutation profiles of two patients with AML with a father-son relationship were obtained at the genetic level, and the son showed a changed mRNA profiles at the gene expression level. The study also identified some new mutation sites in known AML-associated genes, such as FLT3. The present case report may provide novel insight into the molecular events governing the pathogenesis of AML.

\section{Acknowledgements}

Not applicable.

\section{Funding}

No funding was received.

\section{Availability of data and materials}

The datasets used and/or analyzed during the current study are available from the NCBI-SRA repository (https://www. ncbi.nlm.nih.gov/sra/). The BioSample accession number is: SAMN16395638.

\section{Authors' contributions}

SHL and YCL designed the study and wrote the manuscript. SZ, RD, YD and FYF performed all the experiments. SHL, SZ and RD confirm the authenticity of all the raw data. All authors approved the final version of the manuscript.

\section{Ethics approval and consent to participate}

This study has been approved by The General Hospital of Western Theater Command (Ethics approval number: 2016KY82). Informed consent to participate was provided from both participants.

\section{Patient consent for publication}

Not applicable.

\section{Competing interests}

The authors declare that they have no competing interests.

\section{References}

1. De Kouchkovsky I and Abdul-Hay M: 'Acute myeloid leukemia: A comprehensive review and 2016 update'. Blood Cancer J 6: e441, 2016.

2. Tenen DG: Disruption of differentiation in human cancer: AML shows the way. Nat Rev Cancer 3: 89-101, 2003.

3. Rouli DD: Chromosome aberrations in leukemia and lymphoma. Ter Arkh 55: 30-35, 1983 (In Russian).

4. Palanisamy N: Chromosomal translocations in AML: Detection and prognostic significance. Cancer Treat Res 145: 41-58, 2010.

5. Martelli MP, Sportoletti P, Tiacci E, Martelli MF and Falini B: Mutational landscape of AML with normal cytogenetics: Biological and clinical implications. Blood Rev 27: 13-22, 2013.

6. Cancer Genome Atlas Research Network, Ley TJ, Miller C, Ding L, Raphael BJ, Mungall AJ, Robertson A, Hoadley K, Triche TJ Jr, Laird PW, et al: Genomic and epigenomic landscapes of adult de novo acute myeloid leukemia. N Engl J Med 368: 2059-2074, 2013.

7. Ilyas AM, Ahmad S, Faheem M, Naseer MI, Kumosani TA, Al-Qahtani $\mathrm{MH}$, Gari $\mathrm{M}$ and Ahmed F: Next generation sequencing of acute myeloid leukemia: Influencing prognosis. BMC Genomics 16 Suppl 1(Suppl 1): S5, 2015.

8. O'Donnell MR, Tallman MS, Abboud CN, Altman JK, Appelbaum FR, Arber DA, Attar E, Borate U, Coutre SE, et al: Acute myeloid leukemia, version 2.2013. J Natl Compr Canc Netw 11: 1047-1055, 2013.

9. Li H and Durbin R: Fast and accurate short read alignment with Burrows-Wheeler transform. Bioinformatics 25: 1754-1760, 2009.

10. Wang K, Li M and Hakonarson H: ANNOVAR: Functional annotation of genetic variants from high-throughput sequencing data. Nucleic Acids Res 38: e164, 2010.

11. Roberts A, Pimentel H, Trapnell C and Pachter L: Identification of novel transcripts in annotated genomes using RNA-Seq. Bioinformatics 27: 2325-2329, 2011.

12. Trapnell C, Williams BA, Pertea G, Mortazavi A, Kwan G, van Baren MJ, Salzberg SL, Wold BJ and Pachter L: Transcript assembly and quantification by RNA-Seq reveals unannotated transcripts and isoform switching during cell differentiation. Nat Biotechnol 28: 511-515, 2010

13. Trapnell C, Hendrickson DG, Sauvageau M, Goff L, Rinn JL and Pachter L: Differential analysis of gene regulation at transcript resolution with RNA-seq. Nat Biotechnol 31: 46-53, 2013.

14. Reiner A, Yekutieli D and Benjamini Y: Identifying differentially expressed genes using false discovery rate controlling procedures. Bioinformatics 19: 368-375, 2003.

15. Tang H, Wang X, Bowers JE, Ming R, Alam M and Paterson AH: Unraveling ancient hexaploidy through multiply-aligned angiosperm gene maps. Genome Res 18: 1944-1954, 2008. 
16. Lu J, Peatman E, Tang H, Lewis J and Liu Z: Profiling of gene duplication patterns of sequenced teleost genomes: Evidence for rapid lineage-specific genome expansion mediated by recent tandem duplications. BMC Genomics 13: 246, 2012.

17. Aickin $M$ and Gensler $H$ : Adjusting for multiple testing when reporting research results: The Bonferroni vs. Holm methods. Am J Public Health 86: 726-728, 1996.

18. Xie C, Mao X, Huang J, Ding Y, Wu J, Dong S, Kong L, Gao G, Li CY and Wei L: KOBAS 2.0: A web server for annotation and identification of enriched pathways and diseases. Nucleic Acids Res 39(Web Server issue): W316-W322, 2011.

19. Ostrovsky O, Korostishevsky M, Levite I, Leiba M, Galski H, Vlodavsky I and Nagler A: Association of heparanase gene (HPSE) single nucleotide polymorphisms with hematological malignancies. Leukemia 21: 2296-2303, 2007.

20. Mitra AK, Crews KR, Pounds S, Cao X, Feldberg T, Ghodke Y, Gandhi V, Plunkett W, Dolan ME, Hartford C, et al: Genetic variants in cytosolic 5'-nucleotidase II are associated with its expression and cytarabine sensitivity in HapMap cell lines and in patients with acute myeloid leukemia. J Pharmacol Exp Ther 339: 9-23, 2011.

21. Liu CY, Hsu YH, Pan PC, Wu MT, Ho CK, Su L, Xu X, Li Y and Christiani DC; Kaohsiung Leukemia Research Group: Maternal and offspring genetic variants of AKR1C3 and the risk of childhood leukemia. Carcinogenesis 29: 984-990, 2008.

22. te Poele EM, Siedlinski M, Anne de Pagter PJ, Bierings MB, Scherpen FJ, Meeuwsen-de Boer TG, Koppelman GH Postma DS, Kamps WA, Boezen HM and de Bont ES: MBL2 and fever during neutropenia in children with acute lymphoblastic leukaemia. Br J Haematol 157: 132-135, 2012.

23. Cao S, Yang J, Qian X, Jin G and Ma H: The functional polymorphisms of ARID5B and IKZF1 are associated with acute myeloid leukemia risk in a Han Chinese population. Gene 647: 115-120, 2018.

24. Abdel-Wahab O, Mullally A, Hedvat C, Garcia-Manero G, Patel J, Wadleigh M, Malinge S, Yao J, Kilpivaara O, Bhat R, et al: Genetic characterization of TET1, TET2, and TET3 alterations in myeloid malignancies. Blood 114: 144-147, 2009.

25. Petiti J, Rosso V, Lo Iacono M, Calabrese C, Signorino E, Gaidano V, Berger M, Saglio G and Cilloni D: Prognostic significance of The Wilms' Tumor-1 (WT1) rs16754 polymorphism in acute myeloid leukemia. Leuk Res 67: 6-11, 2018.

26. Wu H, Deng J, Zheng J, You Y, Li N, Li W, Wu D and Zhou Y: Functional polymorphisms in the CD44 gene and acute myeloid leukemia cancer risk in a Chinese population. Mol Carcinog 54: 102-110, 2015.

27. Kim B, Kim S, Lee ST, Min YH and Choi JR: FLT3 internal tandem duplication in patients with acute myeloid leukemia is readily detectable in a single next-generation sequencing assay using the pindel algorithm. Ann Lab Med 39: 327-329, 2019.

28. Tien FM, Hou HA, Tsai CH, Tang JL, Chiu YC, Chen CY, Kuo YY, Tseng MH, Peng YL, Liu MC, et al: GATA2 zinc finger 1 mutations are associated with distinct clinico-biological features and outcomes different from GATA2 zinc finger 2 mutations in adult acute myeloid leukemia. Blood Cancer J 8: 87, 2018.
29. Celton M, Forest A, Gosse G, Lemieux S, Hebert J, Sauvageau G and Wilhelm BT: Epigenetic regulation of GATA2 and its impact on normal karyotype acute myeloid leukemia. Leukemia 28: 1617-1626, 2014.

30. Lee JS, Cheong HS, Koh Y, Ahn KS, Shin HD and Yoon SS: MCM7 polymorphisms associated with the AML relapse and overall survival. Ann Hematol 96: 93-98, 2017.

31. Oshrine BR, Olsen MN, Heneghan M, Wertheim G, Daber R, Wilmoth DM, Biegel JA, Pawel B, Aplenc R and King RL: Acquired isochromosome 12p, somatic TP53 and PTEN mutations, and a germline ATM variant in an adolescent male with concurrent acute megakaryoblastic leukemia and mediastinal germ cell tumor. Cancer Genet 207: 153-159, 2014.

32. Foster N, Paulsson K, Sales M, Cunningham J, Groves M, O'Connor N, Begum S, Stubbs T, McMullan DJ, Griffiths M, et al: Molecular characterisation of a recurrent, semi-cryptic RUNX1 translocation $\mathrm{t}(7 ; 21)$ in myelodysplastic syndrome and acute myeloid leukaemia. Br J Haematol 148: 938-943, 2010.

33. Stirewalt DL, Pogosova-Agadjanyan EL, Tsuchiya K, Joaquin J and Meshinchi S: Copy-neutral loss of heterozygosity is prevalent and a late event in the pathogenesis of FLT3/ITD AML. Blood Cancer J 4: e208, 2014.

34. Itzykson R, Kosmider O, Renneville A, Morabito M, Preudhomme C, Berthon C, Adès L, Fenaux P, Platzbecker U, Gagey $\mathrm{O}$, et al: Clonal architecture of chronic myelomonocytic leukemias. Blood 121: 2186-2198, 2013.

35. Cao T, Jiang N, Liao H, Shuai X, Su J and Zheng Q: The FLT3-ITD mutation and the expression of its downstream signaling intermediates STAT5 and Pim-1 are positively correlated with CXCR4 expression in patients with acute myeloid leukemia. Sci Rep 9: 12209, 2019.

36. Schnittger S, Bacher U, Haferlach C, Alpermann T, Kern W and Haferlach T: Diversity of the juxtamembrane and TKD1 mutations (exons 13-15) in the FLT3 gene with regards to mutant load, sequence, length, localization, and correlation with biological data. Genes Chromosomes Cancer 51: 910-924, 2012.

37. Patel RK, Weir MC, Shen K, Snyder D, Cooper VS and Smithgall TE: Expression of myeloid Src-family kinases is associated with poor prognosis in AML and influences Flt3-ITD kinase inhibitor acquired resistance. PLoS One 14: e0225887, 2019.

38. Levis M and Small D: FLT3: ITDoes matter in leukemia. Leukemia 17: 1738-1752, 2003.

39. Sun JY, Mu N, Mu J, Li W, Zhang CG and Wang DM: Expression and significance of PTEN and BCL-2 in acute myeloid leukemia. Zhongguo Shi Yan Xue Ye Xue Za Zhi 26: 121-125, 2018 (In Chinese).

This work is licensed under a Creative Commons Attribution-NonCommercial-NoDerivatives 4.0 International (CC BY-NC-ND 4.0) License. 